\title{
van der Waals interactions of carbon monoxide
}

\author{
Gregory A. Parker \\ parker@nhn.ou.edu \\ Russell T. Pack
}

Follow this and additional works at: https://scholarsarchive.byu.edu/facpub

Part of the Biochemistry Commons, and the Chemistry Commons

\section{Original Publication Citation}

Parker, Gregory A. and Russell T. Pack. "van der Waals interactions of carbon monoxide." The Journal of Chemical Physics 64 (1976): 21-212.

\section{BYU ScholarsArchive Citation}

Parker, Gregory A. and Pack, Russell T., "van der Waals interactions of carbon monoxide" (1976). Faculty Publications. 779.

https://scholarsarchive.byu.edu/facpub/779 


\title{
van der Waals interactions of carbon monoxide*
}

\author{
Gregory A. Parker and Russell T Pack \\ Department of Chemistry, Brigham Young University, Provo, Utah 84602 \\ and Group T-6, University of California Los Alamos Scientific Laboratory, Los Alamos, New Mexico 875457 \\ (Received 9 September 1975)
}

Padé approximant methods and experimental frequency dependent polarizabilities are used to calculate reliable van der Waals $C_{6}$ coefficients for the interaction of $\mathrm{CO}$ with $\mathrm{He}, \mathrm{Ne}, \mathrm{Ar}, \mathrm{Kr}, \mathrm{Xe}, \mathrm{H}, \mathrm{Li}, \mathrm{Na}, \mathrm{K}$, $\mathrm{Rb}, \mathrm{Cs}, \mathrm{H}_{2}, \mathrm{~N}_{2}, \mathrm{O}_{2}, \mathrm{CO}_{2}$, and $\mathrm{CO}$. Error bounds are given for the $\mathrm{C}_{6}$ coefficients and for the $\mathrm{CO}$ oscillator strength sums.

\section{INTRODUCTION AND THEORY}

In a recent paper ${ }^{1}$ (hereinafter called I), one of us calculated van der Waals coefficients for $\mathrm{CO}_{2}$ interactions using the Padé approximant methods of Langhoff and Karplus ${ }^{2}$ to get the contribution to the polarizability from the ultraviolet part of its spectrum and the method of Nir, Adams, and Rein, ${ }^{3}$ which views molecular bands as lines of finite width, to get the infrared contribution. In this paper we use this method to calculate the first reliable van der Waals $C_{6}$ coefficients for interactions involving carbon monoxide, $\mathrm{CO}$.

As is well known, ${ }^{4}$ the van der Waals $C_{8}$ coefficient for the interaction of a $\Sigma$-state diatomic molecule such as $\mathrm{CO}$ with an $S$-state atom takes the form

$$
C_{6}=C_{6}(0)+C_{6}(2) P_{2}(\cos \theta),
$$

where $\theta$ is the angle between the molecular axis and the vector from the center of mass of the molecule to the atom, and $P_{2}$ is a Legendre polynomial. Here each coefficient includes both induction and dispersion contributions:

$$
C_{8}(L)=C_{6}^{1 \text { idd }}(L)+C_{6}^{\mathrm{dis}}(L) .
$$

The induction terms are simply given (in atomic units) by $^{4}$

$$
C_{B}^{1 \mathrm{nd}}(0)=C_{6}^{1 \mathrm{nd}}(2)=\alpha(A) \mu^{2},
$$

where $\mu$ is the permanent dipole moment of the molecule and $\alpha(A)$ is the static dipole polarizability of the atom. Using the experimental value ${ }^{5} \mu(\mathrm{CO})=-0.112 \mathrm{D}$ $=-0,0441 \mathrm{a} . \mathrm{u}$. consistent with the use of experimental polarizabilities, so that the resulting van der Waals potential has been averaged over the ground state vibrational motion, one obtains the $C_{6}^{\text {nd }}(L)$ directly.

If the polarizability anisotropy ratio, $\kappa=\left(\alpha_{11}-\alpha_{1}\right) /$ $\left(\alpha_{11}+2 \alpha_{1}\right)$, is known as a function of frequency, $C_{B}^{d 1 s}(2)$ can be calculated accurately. ${ }^{\circ}$ Unfortunately, for CO values of $\kappa$ have been reported at only two frequencies $\left(\kappa=0.089_{7}\right.$ at $^{7} \omega=0.0720$ a.u. and 0.100 at $^{8} 0.0934$ a. u. ), and their difference is opposite that necessitated by the spectrum of $\mathrm{CO}$ and thus wrong. We assume that the average of the two is the correct value and negligibly different from that at zero frequency. Then, as we have shown elsewhere, ${ }^{4}$ one can obtain reasonable bounds on $C_{6}^{\text {dis }}(2)$ from

$$
C_{B}^{\mathrm{d} 1 \mathrm{~s}}(2)=a_{8} C_{8}^{\mathrm{d} 1 \mathrm{~s}}(0) \text {, }
$$

$$
\frac{\alpha_{11}^{3 / 4}-\alpha_{1}^{3 / 4}}{\alpha_{11}^{3 / 4}+2 \alpha_{1}^{3 / 4}} \leqslant a_{6} \leqslant \kappa,
$$

which gives the estimate

$$
a_{6}=0.083 \pm 0.017
$$

for $\mathrm{CO}$ interactions.

The remaining part of $C_{B}, C_{6}^{\mathrm{dis}}(0)$, is determined in the following section using, with minor modifications, the method of Paper I, which briefly stated is as follows: The experimental frequency dependent polarizability is obtained from refractive index data using the LorenzLorentz equation; then, it is fit using a finite linewidth dispersion term for the ir part and a Cauchy expansion for the uv part. Then, the resulting sum rules (coefficients of the Cauchy expansion) are used to construct upper and lower Padé bounds to the polarizability. From these, effective oscillator strengths and excitation frequencies are obtained from which upper and lower bounds to $C_{6}^{\mathrm{d} 1 \mathrm{~s}}(0)$ are obtained.

\section{CALCULATIONS AND RESULTS}

As input data we used values of the refractive index $\eta$ of $\mathrm{CO}$ at 94 wavelengths ranging from 237. 9-13000 nm reported by Ländolt and Bornstein ${ }^{\theta}$ and the International Critical Tables. ${ }^{10}$ We were unable to find any newer measurements. The frequency-dependent average polarizability $\left[\alpha=\frac{1}{3}\left(\alpha_{11}+2 \alpha_{1}\right)\right]$ was obtained at each frequency from the Lorenz-Lorentz equation ${ }^{3}$

$$
\alpha(\omega)=\frac{3}{4 \pi n}\left(\frac{\eta^{2}-1}{\eta^{2}+2}\right),
$$

where $n$ is the number density of $\mathrm{CO}$ molecules. The Benedict-Webb-Rubin equation of state for $\mathrm{CO}$ of

TABLE I. Oscillator strength sums for CO. The ultraviolet contributions were obtained directly from fitting Eq. (S) and the infrared contributions from expansion of the first term in Eq. (9) after fitting: $S(0)$ is from the Reiche-Thomas-Kuhn sum rule. All are in Hartree atomic units.

\begin{tabular}{rlll}
\hline \hline$k$ & $S^{\text {uv }}(k)$ & $S^{\text {Ir }}(k)$ & $S^{\text {tot }}(k)$ \\
\hline 0 & 14.0000 & $1.37 \times 10^{-5}$ & 14.0000 \\
-2 & $13.0891 \pm 0.0002$ & $0.142 \pm 0.003$ & $13.231 \pm 0.003$ \\
-4 & $47.842 \pm 0.055$ & $1.48 \times 10^{3}$ & $1.53 \times 10^{3}$ \\
-6 & $318.6 \pm 5.7$ & $1.51 \times 10^{7}$ & $1.51 \times 10^{7}$ \\
-8 & $2800 \pm 230$ & $1.53 \times 10^{11}$ & $1.53 \times 10^{11}$ \\
-10 & $26500 \pm 3000$ & $1.59 \times 10^{15}$ & $1.59 \times 10^{15}$ \\
\hline \hline
\end{tabular}


TABLE II. Effective oscillator strengths $f_{n}$, frequencies $\omega_{n}$, and widths $\gamma_{n}$ for $\mathrm{CO}$. Here $A_{n}=f_{n} / \omega_{n}^{2}$. The parameters for $n=1$ were obtained from Eq. (9). On the others $u$ and $l$ label parameters from the upper and lower bounding Pade approximants. Hartree atomic units.

\begin{tabular}{lclll}
\hline \hline & $f_{n}$ & $A_{n}$ & $\omega_{n}$ & $\gamma_{n}$ \\
\hline 1 & $1.36874 E-5$ & 0.1415644 & $9.832942 E-3$ & $3.040752 E-4$ \\
$2 u$ & 13.13886 & 6.919783 & 1.377947 & 0.0 \\
$2 l$ & 5.539610 & 9.959741 & 0.7457883 & 0.0 \\
$3 u$ & 0.8611361 & 6.169317 & 0.3736090 & 0.0 \\
$3 l$ & 0.3271316 & 3.129359 & 0.3233207 & 0.0 \\
\hline
\end{tabular}

Schiller and Canjar ${ }^{21}$ gives as the conversion factor from measurements at STP to $\alpha(\omega)$ in atomic units

$$
3 /(4 \pi n)=59926.98 a_{0}^{3} \text {. }
$$

$\alpha(0)$ can also be obtained from dielectric constant measurements $^{12}$ via the Clausius-Mosotti equation, ${ }^{13}$ and the value $\alpha(0)=13.21 \pm 0.13 a_{0}^{3}$ thus obtained is consis tent with the $\alpha(0)=S(-2)$ determined below from refractive index data but sufficiently uncertain that it could not be used to improve accuracy.

The experimental $\alpha(\omega)$ were then fit by the formula (see Paper I)

$\operatorname{Re} \alpha(\omega)=\frac{A_{2}\left[1-\left(\omega / \omega_{1}\right)^{2}\right]}{\left[1-\left(\omega / \omega_{1}\right)^{2}\right]^{2}+\left(\gamma_{1} \omega / \omega_{1}^{2}\right)^{2}}+\sum_{k=0}^{4} S^{4 r}(-2 k-2) \omega^{2 k}$

where the first term is the contribution of the ir spectrum of $\mathrm{CO}$, and the uv contribution has been expanded in a Cauchy series. The parameters in (9) were determined by an iterative procedure in which the three parameters in the ir term and $S(-10)$ were calculated using a nonlinear flexible tolerance minimization program $^{14}$ which included the Stieltjes constraints, ${ }^{2}$ and $S(-2)$ through $S(-8)$ were calculated using a linear least squares method. This procedure gave a root mean square deviation of $0.041 a_{0}^{3}$, which is half that of the 2 term fit of Nir, Adams, and Rein, ${ }^{3}$
The resulting oscillator strength sums $S^{\mathrm{ur}}(k)$ are given in Table I. These were used to calculate the $[2,1]$ Padé approximants ${ }^{2}$ from which the effective oscillator strengths and effective frequencies of Table II and upper and lower bounds to the $C_{8}^{\text {dis }}(0)$ could be obtained as in Paper I. However, the Padé methods treat the $S^{\text {uv }}(k)$ as though they were exact and had been determined with an infinite number of terms in the summation in Eq. (9). To get an estimate of the additional error thus introduced, we varied $S^{\mathrm{ur}}(-10)$, which is not needed by the $[2,1]$ Padé approximants, over the entire range allowed by the Stieltjes constraints to see what values of the other $S(k)$ and of $C_{B}^{\text {dis }}(0)$ that would generate. That gave the error limits shown in Table I, but because the original data were quite smooth and the uncertainties in the $S(k)$ are quite small, it only widened the error bounds on the $C_{6}^{\text {dis }}(0)$ slightly.

The resulting $C_{6}^{\text {dis }}(0)$ for the interaction of $C O$ with a number of partners (using the sum rules from Refs. 1 and 2 for the other atoms and molecules) are given in Table III. The numbers shown are the means of the best Padé bounds, but the uncertainties include the range of values generated by the uncertainties in the $S(k)$. Also in Table $\Pi$ are the induction and angle-dependent parts of $C_{B}$ for the partners for which Eq. (1)(3) are adequate. The induction terms are very small because the dipole moment of $\mathrm{CO}$ is so small.

Before concluding we note that the $C_{6}$ coefficients discussed herein are only defined within the Born-Oppenheimer approximation, and only the electronic polarizability should be used in calculating $C_{6}^{d 1}(0)$. In a future publication, ${ }^{4}$ we discuss the vibrational coordinate dependence of $C_{6}$ in detail and show that. to a good approximation, the experimental quantities needed in calculating $C_{8}$,are the $S^{\mathrm{ur}}(k)$ rather than the $S^{\text {tot }}(k)$. In the present case the ir spectrum of CO contributes only about $1 \%$ of the static polarizability, and inclusion of the ir contribution ( $n=1$ term of Table $I$ ) would change the $C_{B}^{\text {d1s }}$ of Table III by a completely negligible amount (less than $0.1 \%$ ). In our previous paper on $\mathrm{CO}_{2} \mathrm{C}_{6}$ coef-

TABLE III. Van der Waals $C_{6}$ coefficients for the interaction of $C O$ with various partners. All are in Hartree atomic units $\left(e^{2} a_{0}^{5}\right)$.

\begin{tabular}{|c|c|c|c|c|c|c|}
\hline Partner & $C_{6}^{\mathrm{dis}}(0)$ & $C_{6}^{\mathbf{i n d}}(0)$ & $C_{6}^{\text {tot }}(0)$ & $C_{B}^{\text {diss }}(2)$ & $c_{6}^{i \operatorname{lod}}(2)$ & $C_{6}^{\text {tot }}(2)$ \\
\hline $\mathrm{H}$ & $23.0 \pm 0.9$ & $8.74 E-3$ & $23.0 \pm 0.9$ & $1.92 \pm 0.47$ & $8.74 E-3$ & $1.92 \pm 0.47$ \\
\hline $\mathbf{L i}$ & $201.9 \div 4.1$ & $3.18 E-1$ & $202.2 \pm 4.1$ & $16.8 \pm 3.8$ & $3.18 E-1$ & $17.1 \pm 3.8$ \\
\hline $\mathrm{Na}$ & $246 \pm 16$ & $3.25 E-1$ & $247 \pm 16$ & $20.5 \pm 5.7$ & $3.25 E-1$ & $20.8 \pm 5.7$ \\
\hline K & $363 \pm 24$ & $5.59 E-1$ & $364 \pm 24$ & $30.3 \pm 8.4$ & $5.59 E-1$ & $30.8 \pm 8.5$ \\
\hline $\mathrm{Rb}$ & $412 \pm 28$ & $6.13 E-1$ & $412 \pm 28$ & $34.3 \pm 9.7$ & $6.13 E-1$ & $34.9 \pm 9.7$ \\
\hline Cs & $355 \pm 16$ & $6.92 E-1$ & $356 \pm 16$ & $29.6 \pm 7.5$ & $6.92 E-1$ & $30.3 \pm 7.6$ \\
\hline $\mathrm{He}$ & $11.2 \pm 0.8$ & $2.69 E-3$ & $11.2 \pm 0.8$ & $0.93 \pm 0.27$ & $2.69 E-3$ & $0.93 \pm 0.27$ \\
\hline $\mathrm{Ne}$ & $23.8 \pm 2.5$ & $5.18 E-3$ & $23.8 \pm 2.5$ & $1.98 \pm 0.65$ & $5.18 E-3$ & $1.98 \pm 0.65$ \\
\hline Ar & $78.2 \pm 7.6$ & $2.15 E-2$ & $78.2 \pm 7.6$ & $6.5 \pm 2.1$ & $2.15 E-2$ & $6.5 \pm 2.1$ \\
\hline $\mathbf{K r}$ & $111+12$ & $3.25 E-2$ & $111 \pm 12$ & $9.3 \pm 3.0$ & $3.25 E-2$ & $9.3 \pm 3.1$ \\
\hline $\mathrm{Xe}$ & $192 \pm 25$ & $5.31 E-2$ & $192 \pm 25$ & $16.0 \pm 5.7$ & $5.31 E-2$ & $16.0 \pm 5.7$ \\
\hline $\mathrm{H}_{2}$ & $32.1 \pm 1.5$ & & & & & \\
\hline $\mathrm{N}_{2}$ & $80.7 \pm 6.9$ & & & & & \\
\hline $\mathrm{O}_{2}$ & $62.8 \pm 3.3$ & & & & & \\
\hline $\mathrm{CO}_{2}$ & $128.6 \pm 8.7$ & & & & & \\
\hline $\mathrm{CO}$ & $88.4 \pm 9.7$ & & & & & \\
\hline
\end{tabular}


ficients, ${ }^{1}$ this ir contribution was inadvertently included, and the $C_{6}$ coefficients obtained there should be revised downward by about $1 \%$ (which is still well within the stated uncertainity).

*Work performed in part under the auspices of the USERDA and supported in part at Brigham Young University by the USERDA and the University of California Los Alamos Scientific Laboratory through Subcontract No。 XP5-72554. tPresent address of both authors.

${ }^{1}$ R. T Pack, J. Chem. Phys. 61, 2091 (1974).

${ }^{2}$ P. W. Langhoff and M. Karplus, J. Chem. Phys. 53, 233 (1970); P. W. Langhoff and M. Karplus, J. Opt. Soc. Am. 59, 863 (1969); P. W. Langhoff, J. Chem. Phys. 57, 2604 (1972).

${ }^{3}$ (a) S. Nir, S. Adams, and R. Rein, J. Chem. Phys, 59, 3341 (1973); (b) S. Nir, S. Adams, and R. Rein, Int. J. Quantum Chem. Symp. 6, 295 (1972).

${ }^{4}$ See, for example, R. T Pack, J. Chem. Phys. 64, 1257
(1976).

${ }^{5}$ See references in F. P. Billingsley II and M. Krauss, J. Chem. Phys. 60, 4130 (1974).

${ }^{6}$ P. W. Langhoff, R. G. Gordon, and M. Karplus, J. Chem. Phys. 55, 2126 (1971).

${ }^{7}$ N. J. Bridge and A. D. Buckingham, Proc. R. Soc. London Ser. A 295, 334 (1966).

${ }^{8}$ R. L. Rowell and G. M. Aval, J. Chem. Phys, 54, 1960 (1971).

${ }^{9}$ Ländolt-Bornstein, Zahlenwerte und Functionen (Springer, Berlin, 1962), 6 Auflage, II Band, 8 Teil, pp. 871-889.

${ }^{10}$ International Critical Tables, edited by $\mathrm{E} . \mathrm{W}$. Washburn (McGraw-Hill, New York, 1930), Vol. VII, pp. 6-11.

${ }^{11} \mathrm{~F}$. C. Schiller and L. N. Canjar, Chem. Eng. Proc. Symp. Ser. 49, 67 (1953).

${ }^{12}$ Hans-Joachim v. Braunmühl, Phys. Z. 28, 141 (1927).

${ }^{13} \mathrm{~J}$. O. Hirschfelder, C. F. Curtiss, and R. B. Bird, Molecular Theory of Gases and Liquids (Wiley, New York, 1964), pp. 858-861.

${ }^{14}$ D. M. Himmelblau, Applied Nonlinear Programming (McGraw-Hill, New York, 1972), pp. 458-468. 